\title{
Discapacidad y trabajo. Luchas por el reconocimiento
}

\author{
María Noel Míguez Passada ${ }^{1}$
}

Fecha de recepción: 1 de julio de 2013

Fecha de aprobación: 14 de octubre de 2013

\begin{abstract}
Resumen
La presente ponencia trata de un otro en su situación de discapacidad con relación al trabajo como categoría ontológica del ser. Trata sobre los procesos de interiorización y exteriorización de una alteridad signada por la ideología de la normalidad bajo el discurso de la igualdad. Se intentará deconstruir cómo la situación de discapacidad en su correlato con el trabajo da cuenta de realidades, coyunturas y procesualidades en su universalidad, que se particularizan en este caso en lo normativo, en lo estructural y en lo simbólico. En su singularidad, el juego entre (no) ser y (no) estar, marcan las cotidianidades de sujetos concretos donde la construcción del otro en su alteridad se constituye en la situación de discapacidad como una presencia/ausencia a-hegemónica, más aún cuando este tema es analizado desde la categoría trabajo.
\end{abstract}

Palabras clave: Discapacidad, Trabajo, Reconocimiento

\begin{abstract}
This paper explores the disabled other within the workplace as an ontological category of being. It tackles the internalisation and externalisation processes of an otherness marked by an ideology of the normal constructed under the equality discourse. This work attempts to deconstruct the way disability in the workplace reveals realities, situations and universalising processes which turn normative, structural and symbolically particular ones. In its singularity, the play between being and not being marks daily situations of concrete subjects where the construction of the otherness is given by his/her disability condition as a hegemonic presence/absence.
\end{abstract}

Keywords: disability; work; recognition

1 Doctora en Ciencias Sociales, Investigadora/Profesora Agregada Facultad de Ciencias Sociales, Universidad de la República, Constituyente 1502, Piso 4, Montevideo, Uruguay, mnmiguez@fcs.edu.uy 


\section{Introducción}

El tema de la discapacidad, y en uno de sus ejes en la concreción del trabajo, se ha venido materializando en los últimos años a través de marcos normativos específicamente formulados para la protección de derechos de las personas en situación de discapacidad. En el Uruguay, por ejemplo, a partir de la Ley No 18.651 del año 2010 "Para la protección integral de las personas con discapacidad" (como superadora esencialmente de la Ley No 16.095 del año 1989).

En esta procesualidad normativa aparecen cuestiones a interpelar. Por ejemplo, si su existencia no hace más que distanciar unos y otros, traer como presencias antes ausencias que, en la vía de los hechos, siguen siendo ausencias de la alteridad.

El otro, representado en las personas en situación de discapacidad, continuaría siendo reducido en un doble juego de presencia/ ausencia, donde el deber ser impuesto desde el uno mismo normalizador y hegemónico como imagen de uno mismo, allana su esencialidad, aunque los discursos digan lo contrario. Presencia normativa en los marcos que estipulan sus derechos, por lo general ausencia de hechos que los factibilicen; presencia absoluta del deber ser de la normalidad, ausencia real del ser en la diversidad.

A través de las líneas que se irán trazando en esta ponencia, se intentará ir deconstruyendo la relación ausencia/presencia, uno mismo/otro, para poder ir dando cuenta de la relación personas en situación de discapacidad/personas sin situación de discapacidad y cómo el trabajo como categoría ontológica del ser aparece diluido en la mismidad del uno mismo en su hegemonía.

\section{El otro en la ausencia/presencia de uno mismo. Trabajo y situación de discapacidad}

Con la Modernidad se llega a la era de la producción del otro, del otro como objeto de producción. El otro ubicado en una alteri- 
dad radical, en una singularidad irreductible, se torna peligroso a uno mismo. Como dice Baudrillard, "producir al otro como diferencia implica vivir la alteridad como destino" (1994: 169). Ese otro, en su construida alteridad, pone en jaque la dualidad otro/uno mismo en tanto ser que también es mediado por el trabajo como categoría ontológica.

Así, se reduce tal relación a una linealidad, donde el otro y uno mismo se repelen muchas veces hasta la ausencia del otro, en lugar de ser partes fundantes y en retroalimentación de esta dialéctica; en lugar de saberse, producirse y reproducirse por la mediación del trabajo en su ontología. En este sentido, el otro de este artículo es "invitado" a desandar el camino del uno mismo a través de medidas normativas que, pareciera, vuelven a enviar a ese otro a su extranjería, con la sensación de haberse quedado en el "hospedaje" de la "normalidad" y la inclusión (laboral en este caso).

Aparece el mapa de la mismidad que se instaura como una figura única en la relación de unos y otros. Pareciera haber una única forma de materializar el trabajo a través del empleo, un único modelo homogéneo de trabajador que refiere, justamente, a uno mismo y la mismidad. Este mapa "se vuelve calco" en su producción y reproducción de uno mismo como imagen hegemónica del deber ser. El punto es que hasta el mapa desaparece en su calco, en su correlato de imposición, de deber ser.

Presencia reconocida del otro por uno mismo; y al mismo tiempo la mismidad como centro, como control, como vigilante del otro. Presencia de un trabajador ubicado en una situación de discapacidad que lo distancia de uno mismo, y, en ese vaivén, uno mismo, quien no está ubicado en una situación de discapacidad, le hace de vigilante para que sea lo más silimar a la mismidad. Parecieran no poder reconocerse otras formas de materializar el trabajo, ya que esa materialización no es reconocida por la mismidad si no se hace como tal.

En esta procesualidad dialéctica de uno mismo y otro, se le niega generalmente al otro la posibilidad de ser. Sólo puede ser 
desde el deber ser de uno mismo. Cuando ese otro se disuelve en el deseo arbitrario de uno mismo desaparece como tal, tranformándose de cierta forma en un apéndice de la mismidad. Se disuelve así la potencialidad de un trabajador que en su singular situación de discapacidad queda desustancializado en la mismidad impuesta por uno mismo. Su presencia se torna con el discurso ajeno; su ausencia se celebra bajo el discurso (no los hechos) de la igualdad.

En este sentido, se entiende que la única manera por la que un trabajador en situación de discapacidad es reconocido en su ontología, resulta a través de su "ser como los demás". Su trabajo se torna hegemónico, o al menos así su exigencia para su subsistencia. El otro, en su alteridad, en su distinción, es llevado a esa hegemónica materialización bajo el entendimiento de que es la única manera de hacerlo. Y, así, aparecen leyes específicas, políticas de "discriminación positiva", discursos sobre la inclusión y la igualdad cuando, en los hechos, en la mayoría de las situaciones, no se está más que llevando al otro al terreno de la mismidad más absoluta del uno mismo.

Se considera que diluir al otro en uno mismo implica pasar de una forma a otra; una especie de desaparición. Desaparecer sería diluir las esencias en las apariencias. Al decir de Baudrillard, "en lugar de ser el teatro suntuoso de múltiples formas iniciales de la crueldad y de la versatilidad de las apariencias, lugar de lo fantasmagórico de las especies, de los sexos y de las diversas maneras de morir, el cuerpo no es otra cosa que la exposición de una sola marca entre todas" (1987: 42). Cuerpo del trabajador, tanto de uno mismo como de otro, hacen a la potencial realización de proyectarse como sujeto, de abstraerse, de reificarse, de trascender su cosificación. Ello requiere que las esencias constitutivas de uno mismo y otro se anclen y potencien desde su propio ser, no desde el deber ser del otro por considerarse lo hegemónico. La mismidad en su concreción del empleo empantana y desustancia tanto a otro como a uno mismo puesto que reduce a la sustancia de lo conocido, a la protoforma 
del empleo moderno, imposibilitando la proyección de la emancipación a través del trabajo como categoría ontológica.

La forma de nombrar determina el ser y estar de uno mismo y otro y cómo se materializan en el trabajo. Si desde el lenguaje de los discursos normativos se plantean formas hegemónicas de factibilizar el trabajo de las personas en situación de discapacidad de la manera lo más parecida posible a las que no se hallan en tal situación, se considera que se está reproduciendo la protoforma del empleo en su mismidad. El otro queda así ausente en su esencia y se potencia su presencia a través de cómo logra devenir en la apariencia de trabajador del uno mismo.

Por otra parte, a través del lenguaje, en lo discursivo se puede distorsionar el pensamiento y la imaginación, y reducir al pleno convencimiento de todos de que la protoforma del empleo como única forma de expresión del trabajo es la "verdadera". De esta manera, cualquier reivindicación que intente transgredir esta posibilidad de "empleo para todos" de la mismidad queda en jaque y tildada de irreverente. Se pierde el espacio del pienso, de un traspasado de la protoforma empleo de la mismidad como concreción del trabajo como categoría ontológica del ser.

Si la utopía en la relación discapacidad - trabajo se la remite concretamente a la protoforma empleo de la mismidad como única objetividad posible, la misma pierde su encanto, su horizonte de transformación, su traspasado hacia una nueva objetividad subjetivada. El sujeto objetivo queda cosificado en la apariencia, no sólo del otro, sino también de uno mismo. El sujeto/objeto queda pasivo en los sentidos (Braudillard, 1987).

El otro, lejos de quedar anulado en su alteridad, en su producción y reproducción, comienza a ser espejo interiorizado de uno mismo, de lo distinto que hay que incorporar para no desaparecer del todo. Se anclan las representaciones de uno mismo en el otro, de manera de sortear los fantasmas de ser un otro que no quisiera ser uno mismo. En esta complejidad, el otro es la ausencia/presencia de uno mismo. 


\section{La situación de discapacidad en la protoforma del empleo de la mismidad}

Cuando la forma encontrada para homogeneizar al otro como imagen y espejo de uno mismo, conforme unicidad -Uno- en el todo, resulta la neutralidad del otro a partir de su situación de discapacidad, se considera que se está en un punto de quiebre que urge de retrospectiva, reflexión, pensamiento colectivo a nivel de la sociedad para desnaturalizar tales cuestiones. El camino de la clasificación y calificación en deficiencias, de marcación de la "anormalidad" viene de y reproduce un desconocimiento de unos y otros como sujetos en sociedad.

Pero, ¿cuán peligroso se torna ese otro como para desestabilizar tanto a uno mismo? Skliar (2002), retomando a Jameson, plantea que la construcción del otro maléfico aparece en términos de oposición. El otro siempre termina siendo otro, como algo a ser contenido y normalizado, (re)inventado, institucionalizado... Estereotipia mediante, ¿qué tan otro maléfico puede ser siempre ese otro? Atribuirle maldad al otro (o lo que el otro representa en el imaginario de uno mismo), implica una complejidad a desandar.

Diluir de lo posible cualquier heterogeneidad en la materialización del trabajo a través del empleo parece ser apenas el punto de partida. La protoforma del empleo de la mismidad aparece en su producción y reproducción como única figura que condensa tal heterogeneidad hacia una homogeneidad. El otro queda neutralizado con el convencimiento de que es para generar igualdad, para evitar discriminaciones, etc.; por lo que no hay riesgo para la protoforma del empleo de la mismidad. Claro está que el culpable enunciado es el otro, por ser otro distinto a uno mismo. Se cosifica al otro como objeto a emancipar a través de la protoforma del empleo de la mismidad, donde halla su mayor paradoja.

¿Será el riesgo, el miedo, la posibilidad, la inmanencia de poder llegar a ser otro? El otro no es otra cosa que el espejo de uno mismo, que puede mostrar quien es uno y quien podría ser. La persona 
en situación de discapacidad, con su estado corpóreo disímil al estipulado por una norma estadística, es un otro que irrumpe, que desampara la mismidad, el deber ser.

\section{Luchas por el reconocimiento. Singularizando en Uruguay.}

Como pareciera no haber hoy día otra posibilidad en el horizonte que la protoforma del empleo de la mismidad para la concreción del trabajo como categoría ontológica del ser que condense uno mismo y otro, la manera más factible de llevarlo adelante es a través de la creación de marcos normativos que le den sustento. Sin estos marcos normativos, el otro ni siquiera aparecería como una presencia/ausencia llevada lo más posible a su apariencia hegemónica. Se diluiría en su exclusión absoluta.

Se considera medular dar un paneo general sobre el devenir de la concreción de esta protoforma del empleo de la mismidad en el Uruguay, tomando su singularidad como ejemplo de lo que se pretende explicitar. De esta manera, se enumeran sucintamente las normas del derecho positivo que fueron apareciendo $y$, a su vez, la presentación de algunas de las organizaciones que fueron luchando por el reconocimiento de la temática en la arena política y social.

La procesualidad normativa en el Uruguay en torno a discapacidad y trabajo lleva una historia de un poco más de medio siglo: a) Ley $\mathrm{N}^{\circ} 11.139$ de 1942, para el otorgamiento de facilidades a los "enfermos mentales" en el trabajo; b) Ley $\mathrm{N}^{\circ} 11.781$ de 1951, para la disposición de un régimen de facilidades en el trabajo para enfermos cardíacos; c) Decreto $N^{\circ} 7293$ de 1972, para la creación de la escuela de terapia ocupacional y psiquiátrica; d) Ley $\mathrm{N}^{\circ} 14.106$ de 1973, para la provisión de un 2\% de los cargos de la administración pública por personas en situación de discapacidad; e) Decreto $N^{\circ} 267$ de 1974, para la provisión de cargos de personas con "defectos físicos"; f) Acto Institucional N 9 de 
1979, para jubilaciones por invalidez; g) Decreto $\mathrm{N}^{\circ} 182$ de 1983, para la reglamentación del procedimiento y los órganos competentes para el reconocimiento de la incapacidad para el trabajo o el empleo habitual; h) Ley $\mathrm{N}^{\circ} 15.841$ de 1986, para la modificación del artículo 44 del Acto Institucional $\mathrm{N}^{\mathrm{0}}$ 9, de 23 de octubre de 1979 con relación a las pensiones por invalidez; i) Ley $\mathrm{N}^{\circ}$ 15.878 de 1987, para la aprobación del Convenio OIT 159 sobre la "readaptación profesional y el empleo de las personas inválidas"; j) Ley $\mathrm{N}^{\circ} 16.074$ de 1990, para el seguro por accidentes de trabajo y enfermedades profesionales; k) Ley $\mathrm{N}^{\circ} 16.095$ de 1989, para la protección integral de las "personas discapacitadas"; 1) Decreto $N^{\circ} 373$ de 1993, para la exoneración de aranceles en la importación de aparatos médicos, prótesis y vehículos ortopédicos; m) Ley $\mathrm{N}^{\circ} 16.592$ de 1994, para la interpretación de la Ley $\mathrm{N}^{\circ}$ $16.095 ;$ n) Ley $N^{\circ} 16.713$ de 1995, para la seguridad social (prestaciones sobre vejez, invalidez y sobrevivencia); ñ) Ley $N^{\circ} 16.759$ de 1996, para la modificación de la ley anterior sobre la retención sobre salarios y haberes de pasividades; o) Resolución N² 2.095 de 1997, para la expedición del "Carné de Discapacidad"; p) Decreto $\mathrm{N}^{\circ} 381$ de 1998, para la aprobación de un Baremo para la "valoración del grado de invalidez"; q) Decreto Nº 382 de 1998, para las normas sobre jubilación por incapacidad total; r) Ley $\mathrm{N}^{\circ}$ 16.929 de 1998, para la pensión por invalidez y vejez; s) Ley $\mathrm{N}^{\circ}$ 17.106 de 1999, para las normas de pensión por invalidez o vejez en situaciones de discapacidad severa; t) Ley $N^{\circ} 17.216$ de 1999, para la provisión de vacantes de personas en situación de discapacidad en organismos públicos; u) Ley $\mathrm{N}^{\circ} 17.230$ de 2000, para considerar como actividad productiva la realizada por mayores de 15 años en establecimientos educacionales; v) Ley $\mathrm{N}^{\circ} 17.266$ de 2000, para la "compatiblidad entre la actividad del discapacitado, en cualquier forma pública o privada, con la pensión por invalidez"; w) Ley N 17.847 de 2004, para los topes de pensión por invalidez; x) Resolución N 32-30 del BPS de 2006, para la flexibilización de criterios para el acceso al beneficio de la pensión por invalidez por parte de "discapacitados severos"; y) Ley 
$N^{\circ} 18.094$ de 2007, para la modificación de los conceptos utilizados sobre discapacidad en textos normativos y normas para su ingreso en la función pública; z) Ley N 18.651 de 2010, para la "protección integral de las personas con discapacidad" (CNHD, 2013; Discapacidad Uruguay, 2013).

Uruguay ha ratificado en el año 2008 la Convención de Derechos de las Personas con Discapacidad (CDPD) de Naciones Unidas del año 2006 y ha aprobado su Protocolo Facultativo en el 2011.

En cuanto a las luchas por el reconocimiento existen en el Uruguay organizaciones de la sociedad civil que han trabajado históricamente en la discapacidad, como ser: Unión Nacional de Ciegos del Uruguay (UNCU), Asociación de Sordos del Uruguay (ASUR), Plenario Nacional de Organizaciones de Personas con Discapacidad (Plenadi), Organización Nacional Pro Laboral para Lisiados (ONPLI), Federación Uruguaya de Asociaciones de Padres e Hijos con Capacidades Mentales Diferentes (FUAP), Asociación Down del Uruguay, Movimiento Nacional de Recuperación del Minusválido (MONAMI), Asociación Uruguaya de Padres de Personas con Autismo Infantil (AUPPAI), Asociación de Ciegos de Salto (ACISA), Asociación de Padres y Amigos de personas con Discapacidad de Tacuarembó (APADISTA). Algunas de estas asociaciones hoy día se encuentran representadas en la Comisión Nacional Honoraria de la Discapacidad, a partir de lo estipulado por la Ley No 18.651 de 2010.

En cuanto a las luchas por el reconocimiento en la dualidad uno mismo/otro, éstas se entienden a partir de lo planteado por Honneth en tanto que "... los cambios sociales normativamente orientados son impulsados por las luchas moralmente motivadas de grupos sociales, el intento colectivo de proporcionar la implantación de formas ampliadas de reconocimiento recíproco institucional y cultural" (Honneth, 1997: 115). Las ausencias se tornan presencias cuando las luchas por el reconocimiento anclan sus bases en la superación de la distinción de alteridades, la mayoría de las veces alterida- 
des excluidas. El otro comienza a ser reconocido por uno mismo, y viceversa, en la trascendencia de la mismidad y el encuentro de una nueva forma de (re)conocerse.

Pensadas con relación a la protoforma del empleo de la mismidad, establecida a partir de marcos normativos como lo materializa el devenir presentado, las luchas por el reconocimiento se fueron haciendo carne a través de los movimientos dados por las diversas asociaciones involucradas en la temática de la discapacidad. El punto es que se hagan carne y no callo, como diría Scribano (2007). Que una protoforma del empleo de la mismidad se haga callo implica su cosificación, por lo que más allá de que haya sido conquistada por luchas iniciales por el reconocimiento, luego se cristalizan en más profundos contrastes y "dolor social" entre uno mismo y otro. Las luchas por el reconocimiento implican cambios y superaciones constantes; puntos de partida que se transforman en trampolines para la superación de situaciones anidadas... y anudadas muchas veces.

En su teoría del reconocimiento, Honneth (1997) introduce sistemáticamente la dialéctica y la procesualidad, en tanto se refiere a un reconocimiento recíproco y diferenciado, en el vaivén de lo individual y lo colectivo. En las luchas por el reconocimiento, este autor retoma de Hegel los pilares esenciales para que éstas se materialicen, a saber: el amor, el derecho y la solidaridad.

En la construcción de las relaciones entre uno mismo y otro, confluyen relaciones primarias entre sujetos ubicados en estas distinciones. El amor, según Honneth (1997), retomando a Hegel, debe ser entendido en estas relaciones primarias, ya que resulta el primer espacio donde el reconocimiento aparece como presencia $\mathrm{o}$, en su ausencia, desmaterializa la esencia del otro en su diferencia. Cuando el amor permite el reconocimiento recíproco de uno mismo y otro, ambos se confirman en su naturaleza necesitada. Ya no es el otro que necesita de uno mismo, sino que ambos se reconocen como sujetos de necesidad. El reconocimiento es recíproco. Ya no hay necesidad de destruir (hacerlo ausente) al otro en su 
alteridad, sino todo lo contrario, reconocerlo (hacerlo presente) para superar en el encuentro situaciones de anclaje para el uno mismo y el otro en la mismidad.

En este sentido, la protoforma del empleo de la mismidad, en el caso de cosificarse y no superarse en el devenir, llevaría a la ausencia del otro, a su eliminación, al desencuentro, al desamor $y$, en este vaivén, a la desaparición, también, de uno mismo. Así, "el amor debe concebirse como "un ser-sí-mismo en el otro"" (Hegel apud Honneth, 1997: 118), en tanto situación intersubjetiva de tensiones comunicativas.

El segundo elemento que introduce Honneth (1997) de Hegel es el derecho. En este sentido, se entiende que partiendo del amor como espacio para la afirmación sostenida de la autonomía, y estando ligado al presupuesto individual de la simpatía o atracción por el otro, la ausencia de este estado quedaría librado a voluntades individuales del reconocimiento o no del otro en su diferencia. El derecho aparece como demarcación para la estructuración del reconocimiento más allá de las (in)voluntades individuales. De esta manera, "sólo desde la perspectiva normativa de un "otro generalizado" podemos entendernos a nosotros mismos como personas de derecho, en el sentido que podemos estar seguros de la realización social de determinadas de nuestras pretensiones" (Honneth, 1997: 132). El derecho, en tanto "especifica constitución de las relaciones de derecho modernas", se introduce como una forma de reconocimiento recíproco, bajo el supuesto y el convencimiento que todos los seres son iguales y libres. De esta manera, "en el Estado el hombre es tratado y reconocido como ser racional, como libre, en tanto que persona; y el singular se hace merecedor de este reconocimiento, porque por el sobrepasamiento de su conciencia de sí natural, se somete a una generalidad, a la voluntad en sí y para sí, a la ley, por consiguiente se porta frente a los otros en una forma con validez general, los reconoce por lo que él mismo quiere valer en tanto que libre y persona" (Hegel apud Honneth, 1997: 133).

Se considera que en la universalidad hegeliana, el trabajo en su abstracción halla su "verdad" como absoluto. Es en la parti- 
cularidad que encuentra la construcción de protoformas que lo anidan en anclajes que pueden ir hacia la presencia o la ausencia del otro en la alteridad con relación a uno mismo y la protoforma del empleo de la mismidad. Pero esa protoforma del empleo de la mismidad no es otra cosa que la cosificación devenida en un momento histórico dado, a partir de luchas (o ausencia de éstas) por el reconocimiento.

Los marcos normativos encarnados en el derecho positivo permiten ir dando cuerpo a un devenir que requiere procesualidad para no terminar eliminando al otro en su ausencia con el discurso de su presencia y la imagen de uno mismo. En la teoría del reconocimiento, no debería haber espacio para la ausencia del otro, en tanto "del reconocimiento de la persona como tal se diferencia la valoración de un hombre, ante todo porque en ella no se trata de la aplicación empírica de normas intuitivamente sabidas, sino de la valoración gradual de cualidades y capacidades concretas" (Honneth, 1997: 139).

A partir de las leyes que hoy día cobran vigencia en Uruguay, se entiende que en la Ley 18.651 del año 2010 de Uruguay, queda anclada en las conceptualizaciones clasificadoras y calificadoras del otro en su singularidad como responsable de su situación individual, sin reconocer que esta situación está siendo vista con la mirada de uno mismo y la reproducción de la mismidad. Por eso, se considera que se hace necesario superar los contenidos de carácter normalizador, de readaptación, de rehabilitación que esta ley plantea ${ }^{2}$. En esta normatividad, aparecen importantes distin-

2 En su Artículo 2, la Ley $\mathrm{N}^{\circ} 18.651$ del Uruguay define: "Se considera con discapacidad a toda persona que padezca o presente una alteración funcional permanente o prolongada, física (motriz, sensorial, orgánica, visceral) o mental (intelectual y/o psíquica) que en relación a su edad y medio social implique desventajas considerables para su integración familiar, social, educacional o laboral" (Ley $\mathrm{N}^{\circ} 18.651$, 2010: 1). Términos como "padezca", "presente", marcan sufrimiento y singularización en la persona como portadora de un estado (o falta del mismo); la clasificación por deficiencias, se considera que no hace más que continuar reproduciendo modelos médicohegemónicos perimidos y ampliamente superados en muchos lugares del mundo. Se cree que esto no se da porque se trate de países más o menos "desarrollados", 
ciones entre uno mismo y otro, entre la reivindicación absoluta de la mismidad bajo el discurso de la diversidad y la real inclusión del otro en pro de la igualdad.

Volviendo sustancialmente a los argumentos de la teoría del reconocimiento, aparece una cuestión que podría poner en jaque el tema del derecho positivo para sujetos libres e iguales con relación a la temática en cuestión. Y es el tema de la autonomía. A partir de un enfoque racional, las propiedades atribuidas a una persona pueden cambiar conforme a lo dispuesto por el derecho. En el caso de demarcar a un otro como "incapaz" (normativamente desde el derecho positivo; normalidad mediante por la ideología), se le está quitando su capacidad de ser autónomo. El punto es que la condición de "incapaz" dada al otro es atribuida por uno mismo. Las líneas demarcatorias comienzan a hacerse tenues cuando las delimitaciones con el discurso de la objetividad terminan siendo siempre subjetivas. El afán categorizador y clasificador de la ideología de la normalidad, que enaltece y hace Uno a uno mismo y otro, llevaría a la ausencia del otro en su condición de sujeto, por su presencia etiquetada de "incapacitado", "discapacitado", "inválido"... Se le estaría quitando al otro la posibilidad de "participar conforme a derecho" (Honneth, 1997: 140).

Se entiende que las luchas por el reconocimiento llevadas adelante por diversas asociaciones de la sociedad civil así como los cambios en las percepciones en la arena política, han ido permitiendo devenir en marcos normativos que fueron haciendo presentes al otro en distintos momentos. De ahí la cantidad que pareciera excesiva de leyes, decretos, etc., que le han ido dando procesualidad a la temática de la discapacidad en torno al trabajo.

sino por la forma de pensar y pensarse en torno a la discapacidad, las sensibilidades devenidas, las representaciones que se tienen de ese otro en su alteridad y la autopercepción de ese otro como imagen de uno mismo. 
La tercera dimensión a la que alude Honneth (1997), retomando a Hegel, para su teoría del reconocimiento, es la solidaridad. La misma la entiende como la necesidad de todo ser humano de ser valorado socialmente con relación a sus cualidades y facultades concretas, más allá de si éstas estén contempladas en el amor (como dimensión más subjetiva y fluctuante) y en el derecho (como reconocimiento jurídico objetivo, pero también discursivo). A esta relación recíproca de reconocimiento dada por la solidaridad, Hegel la denomina "eticidad": "...mientras que el derecho moderno presenta un medio de reconocimiento que expresa la propiedad general de los sujetos en forma diferenciada, esa segunda forma de reconocimiento requiere un médium social que puede expresar la diferencia de cualidad entre los sujetos humanos en una forma intersubjetivamente coaccionante. Esta tarea de mediación efectúa en el plano social un marco de orientación simbólicamente articulado y siempre abierto y poroso, en el que se formulan los objetivos y valores éticos, cuyo conjunto constituye la evidencia cultural de una sociedad" (Honneth, 1997: 149). En este sentido, el valor social determinado es medido a partir del grado por el que se contribuye a la realización de los objetivos sociales.

Se considera que en la protoforma del empleo de la mismidad de la actualidad, el otro termina por lo general siendo construido e inventado como algo ajeno a su esencia. Al otro construido en una situación de discapacidad le urge, según la mismidad, recorrer y transitar el camino de la patologización - clasificación - distinción - etiquetamiento. Es un nunca-otro y siempre-otro. Nunca-otro en tanto existe por haber sido inventado y ubicado en su alteridad; siempre-otro como otro de uno mismo, como imagen, espejo, (des)semejanza de la mismidad.

En estas (re)ubicaciones del otro como alteridad, de su clasificación y calificación, aparece el tema de las etiquetas. Para acceder a un empleo contemplado por la protoforma de la mismidad y particularizado en el marco normativo, las personas en situación de discapacidad deben registrarse como tales (en la 
Comisión Nacional Honoraria de la Discapacidad -CNHD- en Uruguay) y obrar en consecuencia.

Se considera que la solidaridad como pilar sustancial de la teoría del reconocimiento, en este sentido, estará garantizada en una sociedad que no sólo defina discursivamente como autónomos a todos los sujetos que la integren, sino haciendo efectiva tal autonomía para una real igualdad y libertad. Se cree que aquí se entra en contradicción justamente con la cuestión de etiquetar, de demarcar, de (de)limitar al otro de uno mismo.

El amor y el derecho contenidos y superados dialécticamente en la noción de solidaridad, dan espacio al encuentro de uno mismo y otro más allá de la mismidad. En este caso concreto, en la protoforma del empleo de la mismidad. Las sociedades deberían poder no sólo discursivamente reconocerse como solidarias, sino serlo fácticamente. De esta manera, se enaltece la presencia del otro como parte de la dualidad y se aleja la posibilidad de ausencia de ese otro por la homogeneización de uno mismo en la mismidad.

\section{Reflexiones finales}

Las personas en situación de discapacidad requieren ser pensadas y ubicadas en un espacio multihomogéneo, en un espacio donde clasificación y calificación del otro no sean parte de la cotidianidad fundante de la relación entre uno mismo y otro. Donde su condición humana de sujetos no sea adormecida por una espacialidad humana gobernada por personas que desde su situación de no discapacidad imponen su mismidad como la totalidad del deber ser para el otro y uno mismo. En un reconocimiento, como diría Skliar (2002), de las "espacialidades de la/s diferencia/s".

Entonces, ¿por qué no pensar una construcción societal donde uno mismo / otro sean traspasados de la mismidad hacia formas del trabajo que trasciendan la concreción hegemónica del empleo como único potencial de emancipación e igualdad de los sujetos? 
Habrá que continuar pensando reflexivamente por qué urge hoy día construir a ese otro ubicado en una situación de discapacidad por una clasificación corpórea, con relación a la mismidad que la ha producido. Quizá un punto interesante para continuar con esta madeja se halle en las palabras de Deleuze, cuando dice: "Y es que las nociones necesarias para la descripción del mundo (...) permanecerán vacías e inaplicables, si el "otro" no estuviera ahí, expresando mundos posibles" (apud Skliar, 2002: 85).

\section{Bibliografía}

\section{Libros:}

Baudrillard, J. (1987). L'autre par lui-même. París: Galilée.

Baudrillard, J. et Gillaume, C. (1994). Figures de l'altérité. París: Descartes $\&$ Cie.

Blanc, A. (1999). Les handicapés au travail. París: Dunod.

Cross-Courtiel, M.L. (1985). Estudio de caso: clasificación legal de las personas discapacitadas en Francia. En: MOOM, W. (1985). Discapacidad y Trabajo. París: Enciclopedia en Salud y Seguridad en el Trabajo.

Ebersold, S. (2001). La naissance de l'inemployable. Ou l'insertion aux risques de l'exclusion. Rennes: Presses Universitaires de Rennes.

HAMONET, C.L. (1990). Les personnes handicapées, París, PUF.

SARTRE, J.P. (1985). Critique de la Raison dialectique. París: Gallimard.

STIKER, H-J. (1982). Corps infirmes et sociétés. París: Aubier-Montaigne.

STIKER, H-J. (2010). Les métamorphoses du handicap de 1970 à nos jours. Soi-même avec les autres. Grenoble: Presses universitaires de Grenoble.

\section{Revistas:}

SCRIBANO, A. (2007). ¡Vete tristeza... Viene con pereza y no me deja pensar!... Hacia una sociologia del sentimiento de impotencia. En: 
LUNA, R. y SCRIBANO, A. (Comp.). Contigo aprendí... Estudios sociales de las emociones. CEA-CONICET-Universidad Nacional de Córdoba-CUSCH- Universidad de Guadalajara. Córdoba. pp. 21-42.

SKLIAR, C. (2002). Pedagogías y alteridades o... ¿y si el otro no estuviera ahí? Educação \& Sociedade, ano XXIII, no 79. pp. 85-124.

STIKER, H-J. (2009). Comment nommer les défiances? Ethnologie française, 2009/3 Vol. 39, p. 463-470. DOI: 10.3917/ethn.093.0463.

\section{Fuentes documentales:}

CAT-Unapei (2013). http://www.cat-unapei.org/que_cat_index.php Archivo revisado 10-01-2013.

CNSA (2013). http: / / www.cnsa.fr/ Archivo revisado 15-01-2013.

COMISIÓN NACIONAL HONORARIA DE LA DISCAPACIDAD. Apartado Legislación. http://www.cnhd.org/legislacion. htm Archivo revisado 17-02-13.

DECRETO PRESIDENCIAL (2006). Se retoma el Artículo $N^{\circ} 42$ de la Ley $N^{o} 16.095$ en la redacción dada por la Ley $N^{o} 17.216$. Uruguay: Poder Legislativo, octubre de 2006.

DISCAPACIDAD URUGUAY (2013). Portal de la discapacidad en el Uruguay. http://www.discapacidaduruguay.org/index.php/ servicios/legislacion Archivo revisado el 17-02-13.

HANDIPOLE (2013). http://www.handipole.org/ Archivo revisado 15-01-2013.

International classification of impairments, disabilities, and handicaps, a manual of classification relating to the consequences of diseases, ICIDIH (1980). Genève: World Health Organisation.

LEY No 16.095 (1989). Sistema de Protección Integral a las Personas Discapacitadas. Uruguay: Poder Legislativo.

LEY N 17.216 (2006). Modifica Art. 42 de la ley $N^{\circ} 16.095$ (4\% cuota de reserva). Uruguay: Poder Legislativo.

LEY No 18.651 (2010). Protección Integral de los Derechos de las Personas con Discapacidad. Uruguay: Poder Legislativo.

LOI n 57-1223 du 23 novembre 1957 sur le reclassement professionnel des travailleurs handicapés. 
LOI n 75-534 du 30 juin 1975 d'orientation en faveur des personnes handicapées.

LOI N²005-102 du 11 février 2005 pour l'égalité des droits et des chances, la participation et la citoyenneté des personnes handicapées.

Ministère des Affaires Sociales et de la Santé. (2013). http:/ /www.social-sante.gouv.fr/ Archivo revisado 15-01-2013.

NU (2006). Convención sobre los Derechos de las Personas con Discapacidad. Washington: ONU.

OMS (2001). Classification Internationale du Fonctionnement, du handicap et de la santé (CIF). Organisation Mondiale de la Santé: Genève.

UNAPEI (2013). http://www.unapei.org/article/la-sous-traitanceaux-structures.html Archivo revisado 15-01-2013. 